\title{
EFECTOS COGNITIVOS DE LA RADIOTERAPIA EN GLIOMAS DE BAJO GRADO
}

\section{COGNITIVE EFFECTS OF RADIOTHERAPY IN LOW GRADE GLIOMAS}

\author{
Ana Sanz', María Eugenia Olivares ${ }^{2}$ y Juan Antonio Barcia ${ }^{3}$ \\ ${ }^{1}$ Fundación Instituto Madrileño de Oncología, Grupo IMO. Servicio de Neurocirugía Fundación Investigación \\ Biomédica Hospital Clínico San Carlos \\ 2 Servicio Ginecología y Obstetricia Hospital Clínico San Carlos. Facultad de Psicología UCM. \\ ${ }^{3}$ Servicio Neurocirugía Hospital Clínico San Carlos. Facultad de Medicina UCM.
}

\section{Abstract}

El aumento en las tasas de supervivencia de los pacientes con gliomas de bajo grado está suscitando un elevado interés en los efectos neurotóxicos asociados a los tratamientos, en especial a la radioterapia. En la actualidad, se señala la presencia de alteraciones principalmente en memoria visual asociada a dicha técnica de tratamiento, aunque existen datos contrarios que postulan que la radioterapia, al igual que otros tratamientos, favorece el control de la enfermedad y preserva el rendimiento neuropsicológico de esta población. No obstante, las limitaciones metodológicas de estos estudios y la dificultad en el seguimiento de estos pacientes podrían estar provocando esta divergencia en los resultados hallados en este campo de investigación. Por ello, se reitera la necesidad de realizar estudios que utilicen las técnicas de tratamiento de radioterapia actuales, que aumentan la seguridad y disminuyen los efectos secundarios neurológicos, así como que apliquen cuestionarios con una adecuada sensibilidad a los efectos neurotóxicos de los tratamientos adyuvantes.

Palabras claves: Gliomas bajo grado, radioterapia, rendimiento neuropsicológico.

\section{Abstract}

The increased survival of patients with low grade gliomas generated increased interest in the neurotoxic effects associated with treatment, especially radiotherapy. Currently, the radiotherapy is associated with visual memory failures in this population. On the contrary, some authors postulate that radiotherapy, as well as other treatments, helps control the disease and preserves the neuropsychological performance. However, methodological limitations of these studies and the difficulty in monitoring these patients could be causing this divergence in the results found in this research field. Therefore, we note the need for studies that use radiation therapy treatment techniques current, which increase safety and reduce neurological side effects, and apply appropriate questionnaires with a sensitivity to the neurotoxic effects of adjuvant treatments.

Key words: Low grade gliomas, radiotherapy, neuropsychological performance.

\section{Correspondencia:}




\section{INTRODUCCIÓN}

La metodología utilizada para la realización del presente artículo de revisión bibliográfica ha sido la elección de low grade gliomas and cognitive deficits or cognitive aspects, como palabras claves de búsqueda, en las bases de datos de Pubmed y EMBASE. Además, se ha consultado en Cochrane library la existencia de evidencia científica al respecto y en las revistas de acceso libre de Biomed.

Los tumores primarios del Sistema Nervioso Central (SNC) representan el $2 \%$ de todas las patologías oncológicas ${ }^{(1)}$. Los gliomas son el subtipo más frecuente ya que constituyen el $50 \%$ de todos los casos, seguidos por los meningiomas (15-20\%). La tasa de incidencia en Estados Unidos para los gliomas en adultos por 100.000 hab./año es de 11 a 12 casos, siendo las cifras similares en España de 8,73/100.00 en varones y 5,4/100.000 en mujeres ${ }^{(2)}$.

La estadificación de los gliomas realizada por la Organización Mundial de la Salud $(\mathrm{OMS})^{(3)}$, los clasifica en función de sus características histológicas, diferenciándolos en gliomas de bajo grado (I-II) y gliomas de alto grado (III-IV): a) grado I incluye lesiones de bajo potencial proliferativo, una naturaleza frecuentemente discreta y una elevada posibilidad de curación posterior a la resección quirúrgica; b) grado II incluye lesiones, por lo general, de carácter infiltrante y de baja actividad mitótica, con elevada probabilidad de recurrencia y con tendencia a avanzar a lesiones con mayor grado de malignidad, y c) grado III y IV, suponen lesiones con una elevada actividad mitótica, capacidad de infiltración claramente expresada y anaplasia, siendo la necrosis una característica exclusiva de los gliomas de grado IV.

Los gliomas de grado II (bajo grado), en los que se centrara este artículo, son tumores de carácter infiltrante, difuso e histológicamente se dividen en astrocitomas, oligodendrogliomas y tumores mixtos ${ }^{(3)}$. Los primeros se caracterizan por presentar la proteína Gliofibrilar ácida positiva y son los más frecuentes. Los oligodendrogliomas representan únicamente el 10\% de este tipo de tumores y se definen por presentar un halo perinuclear $y$, en último lugar, los tumores mixtos muestran células de los dos tipos anteriores.

Este tipo de lesiones comprenden del 20 al 30\% de todos los tumores del SNC, con una prevalencia de 2/100.000 hab. en EEUU y en Europa ${ }^{(4)}$. No obstante, en los últimos años se ha observado un aumento en la incidencia de estas lesiones y una mayor precisión en su diagnóstico, debido a los avances en las técnicas de neuroimagen. Estos tumores suelen aparecer entre la segunda y la cuarta década de vida, con una media de edad en adultos de 35 años y con una tendencia de mayor incidencia en los varones ${ }^{(5)}$. La media de supervivencia de estas neoplasias oscila entre los 6 y 8 años, ya que suelen progresar hacia otros gliomas de mayor malignidad, pudiendo alcanzar los 10 años en el caso de los oligodendrogliomas. Así, el pronóstico de este tipo de tumores está asociado a la edad, el estado general (valorado mediante el índice de Karnofsky), el volumen de la lesión, la invasión de la línea media del cerebro y su histología ${ }^{(6,7)}$. En los últimos años, la literatura está comenzando a considerar el estado neuropsicológico de los pacientes como un factor pronóstico, observándose un mayor riesgo de morbimortalidad en pacientes con deterioro cognitivo moderado desde las primeras fases de la enfermedad ${ }^{(8,9)}$.

Las manifestaciones clínicas más frecuentes son: a) crisis epilépticas, presentes entre el $72 \%$ y el $89 \%$ de los pacientes ${ }^{(10-17)}$; b) aumento de la presión intracraneal, que cursa con dolores de cabeza y náuseas en los momentos iniciales $(10 \%-40 \%)^{(11,15,17)}$; c) cambios en el estado mental general valorados mediante pruebas de screening 
de deterioro cognitivo ( $3 \%-30 \%)$ en el momento del diagnóstico ${ }^{(10,15,17)}$, y d) déficits neurológicos $(3 \%-30 \%)^{(12,15)}$.

En relación a los síntomas neuropsicológicos iniciales, existe escasa información sobre la naturaleza de los mismos, ya que para ello es necesario realizar una valoración exhaustiva previa a la aplicación de cualquier tratamiento médico. Los resultados no son concluyentes, informándose de alteración en algún dominio cognitivo en la mayor parte de los estudios. De esta forma, pueden evidenciarse fallos en el funcionamiento psicomotor ${ }^{(18)}$, en velocidad de procesamiento de la información ${ }^{(19,20)}$, en atención selectiva y dividida ${ }^{(20)}$, así como alteración en nominación verbal y en memoria inmediata visual(19). En función del hemisferio afecto, Yoshii et al. ${ }^{(21)}$ señalaron que los pacientes con gliomas en el hemisferio derecho no presentaban alteraciones neuropsicológicas, mientras que los pacientes con afectación en el hemisferio izquierdo mostraban una alteración cognitiva global y difusa.

En relación al tratamiento de los gliomas de bajo grado, la estrategia terapéutica a seguir suscita muchas controversias debido a la neurotoxicidad de estos; mientras algunos clínicos plantean un tratamiento quirúrgico inmediato con la máxima exéresis posible, seguida de radioterapia (RT) inmediata, otros plantean una política de observación $^{(2,4,6)}$. Es necesario considerar que en estas lesiones es frecuente la utilización de tratamientos adyuvantes, al no poder realizarse una resección completa, siendo la RT la estrategia más frecuentemente empleada aunque el momento temporal de su aplicación no está exento de polémica. Actualmente, en función de los estudios retrospectivos, se ha determinado la dosis de irradiación para este tipo de lesión intracraneal, que oscila entre 45-50 Greys (Gy), con un fraccionamiento máximo de 2 Gy por sesión ${ }^{(22)}$. No obstante, la European Organization for Research and Treatment of Cancer (EORTC) no recomienda su utilización de forma precoz en pacientes con gliomas de bajo grado, excepto en pacientes mayores de 40 años o en personas con lesiones irresecables o con déficit neurológico severo, ya que su utilización no incrementa la supervivencia y sí presenta una elevada tasa de toxicidad neurológica.

En este sentido, la comunidad científica $^{(23)}$ ha identificado los efectos neurotóxicos de la RT en pacientes con tumores cerebrales, dividiéndolos en tres tipos de acuerdo a su momento de aparición y a los mecanismos fisiopatológicos implicados:

a) Efectos agudos: aparecen inmediatamente después de la primera sesión de tratamiento, presentan un carácter transitorio y se asocian al edema vasogénico, secundario a los cambios inducidos por la radiación en la barrera hematoencefálica. Las manifestaciones clínicas más comunes son la letargia, cefalea, vómitos y fiebre.

b) Efectos inmediatos-diferidos: pueden presentarse entre las seis semanas y los seis meses posteriores a la finalización del tratamiento. Aunque se desconocen los mecanismos fisiopatológicos, se cree que pueden estar relacionados con el edema cerebral y la desmielinización de la sustancia blanca. Se caracterizan por un empeoramiento de los déficits neurológicos previos y síntomas similares a los efectos agudos. A nivel cognitivo, se puede producir un deterioro transitorio que consiste en déficit atencionales y de memoria a corto plazo. Suelen presentarse de forma transitoria y su mejoría sucede de forma espontánea entre la primera y sexta semana posteriores a su aparición.

c) Efectos retardados: pueden aparecer desde los seis meses hasta años después de la finalización del tratamiento. Estos presentan un carácter permanente y más severo que los anteriormente citados, pudiendo tomar forma de radionecrosis focal, leuco-encefalopatía y atrofia cerebral. Los 
pacientes pueden presentar un deterioro insidioso y progresivo en atención y memoria, junto con signos neurológicos como por ejemplo demencia e incluso la muerte. En algunos estudios ${ }^{(24-26)}$, se pone de manifiesto una relación entre las puntuaciones en las pruebas neuropsicológicas y la presencia de alteraciones estructurales, en las pruebas de neuroimagen (atrofia cerebral y leuco-encefalopatía).

\section{EFECTOS COGNITIVOS DE LA RADIOTERAPIA EN GLIOMAS DE BAJO GRADO}

Dentro de la Neurooncología, el estudio de los efectos cognitivos de la RT en pacientes con gliomas de bajo grado ha suscitado un elevado interés en los últimos años, ya que la toxicidad relacionada con el tratamiento es un aspecto que preocupa por los siguientes motivos: aumento significativo de la supervivencia de estos pacientes, su impacto en la calidad de vida e implicación en el estado funcional $^{(9,14,27-30)}$. Tal y como se ha indicado, los pacientes con estas lesiones tienen una edad media de 35 años en el momento del diagnóstico, lo que supone que son personas jóvenes, en activo a nivel laboral, familiar y social. Además, los datos obtenidos mediante la aplicación de los cuestionarios neuropsicológicos aportan una información adicional sobre la situación clínica de los pacientes, con respecto a las pruebas de neuroimagen y los exámenes neurológicos, lo que permite aplicar terapias de soporte que favorezcan la calidad de vida de los mismos. En la actualidad, los efectos cognitivos asociados a los tratamientos se están comenzando a considerar en las guías de actuación clínica de los gliomas de bajo grado (EFNS-EANO task force), como un indicador de la efectividad de las técnicas terapéuticas aplicadas ${ }^{(31)}$.

A pesar de la relevancia de este área de conocimiento, se ha encontrado un esca- so número de investigaciones, aproximadamente veinte estudios, debido a la baja prevalencia de estas lesiones y a la dificultad clínica y metodológica en la recogida de la información neuropsicológica. Como puede observarse en la Tabla 1, los datos en este área son discrepantes, probablemente por la metodología aplicada en cada uno de los estudios, pudiéndose identificar dos tipos de resultados:

1. Aquellos que sí refieren la presencia de déficits o alteraciones cognitivas asociadas a la RT, principalmente en memoria visual, describiendo variaciones en el rendimiento cognitivo en los cinco años posteriores a la finalización del tratamiento. Además, en estas investigaciones se ha evidenciado correlación directa entre las puntuaciones neuropsicológicas (atención, velocidad procesamiento información, memoria y funciones ejecutivas) y la presencia de atrofia cerebral y leucoencefalopatía, en las pruebas de neuroimagen ${ }^{(24-26)}$.

2. En otros estudios, se señala la ausencia de afectación neuropsicológica asociada a la aplicación de este tratamiento. En este sentido, algunos autores consideran que las alteraciones neuropsicológicas observadas se asocian principalmente al tumor. Asimismo, señalan que la RT, al igual que otros tratamientos, puede proporcionar una mejoría o estabilización del estado del funcionamiento neuropsicológico, debido al control que se ejerce sobre la enfermedad ${ }^{(9,32,33)}$.

\section{DATOS A FAVOR DE LA EXISTENCIA DE DETERIORO COGNITIVO ASOCIADO A LA RADIOTERAPIA}

En este apartado pueden diferenciarse tres tipos de estudios, aquellos que comparan el rendimiento neuropsicológico de pacientes con gliomas de bajo grado que reciben tratamiento de $\mathrm{RT}$, con respecto a aquellos que no lo reciben $y / o$ que valoran dicho rendimiento a lo largo del tiempo, 
es decir, antes de recibir la RT y en diferentes momentos posteriores a la finalización de dicho tratamiento; el segundo tipo de estudios, comparan el funcionamiento cognitivo de acuerdo a la modalidad de tratamiento de RT aplicado (RT holocraneal vs. RT focal) y el tercer tipo, lo hacen de acuerdo a la dosis total y fraccionada de radiación recibida. Por último, se citan otros factores que se asocian con el deterioro cognitivo producido por la RT.

\section{a) Rendimiento neuropsicológico en función de recibir o no tratamiento de radioterapia en pacientes con gliomas de bajo grado}

Unos de los principales autores en este campo son Correa et al. ${ }^{(34,35)}$, que Ilevaron a cabo algunas de las investigaciones relevantes con una importante repercusión científica, destacándose dos estudios. El primero es de carácter transversal, con un momento de medida a los de 3 años y medio del fin de tratamiento; mientras que el segundo estudio es longitudinal y prospectivo, con una valoración neuropsicológica previa a la aplicación de la RT y un seguimiento máximo de doce meses después de su finalización. En ambas investigaciones, estos autores valoraron un amplio número de dominios neuropsicológicos mediante cuestionarios con cualidades psicométricas óptimas ${ }^{(36)}$.

En el primer estudio ${ }^{(34)}$, los autores concluyeron que los pacientes que recibían RT presentaban exclusivamente un peor rendimiento en el dominio de memoria visual, con respecto a los pacientes con la misma lesión que no recibieron este tratamiento adyuvante. Además, señalaron que los tratamientos anti-epileptógenos se asociaban con peores puntuaciones en los cuestionarios de funciones ejecutivas y de funcionamiento psicomotor.

En el segundo estudio, Correa et al.(35), compararon el rendimiento neuropsicoló- gico de un grupo de pacientes que recibió $\mathrm{RT}$, con respecto a pacientes con gliomas de bajo grado que no recibieron dicho tratamiento. Estos autores, informaron que los pacientes que recibían RT mostraban déficits en el funcionamiento psicomotor, previamente a la aplicación de este tratamiento. Además, definieron el patrón de rendimiento neuropsicológico de los pacientes con gliomas de bajo grado a lo largo de un año. Todos los pacientes mostraron una mejoría en este funcionamiento en los seis primeros meses posteriores al tratamiento, seguida de un declive cognitivo global (que no conllevaba alteraciones clínicas) doce meses después de la finalización del mismo, en ambos grupos. En último lugar, se realizó un seguimiento más largo en el tiempo a un grupo reducido de pacientes (aquellos que quisieron participar), observándose en el grupo que recibió $\mathrm{RT}$ resultados que ponían de manifiesto un deterioro clínico en recuerdo visual y en algunos componentes de las funciones ejecutivas; mientas que evidenciaron una mejoría en fluidez fonológica, memoria de trabajo y en calidad de vida, en los pacientes que no recibieron dicha modalidad terapéutica.

Otro trabajo digno de mención en esta área, es el realizado por Postma et al. ${ }^{(25)}$, cuyo objetivo principal fue determinar la presencia de atrofia cerebral y alteraciones en la sustancia blanca asociadas a la RT, así como comparar el rendimiento neuropsicológico de pacientes que recibieron RT, con respecto a aquellos que no lo hicieron. Para ello, valoraron variables de memoria y atención, que incluyó la velocidad de procesamiento de la información como dominio atencional, en pacientes con gliomas de bajo grado cuatro años después de la finalización del tratamiento de RT. Así, más de la mitad de los pacientes que recibieron esta modalidad terapéutica presentaban estas alteraciones en las pruebas de neuroimagen (61\%), en 
comparación al 6\% de los pacientes que no habían recibido tratamiento. Por otro lado, la presencia de atrofia cerebral se asociaba con una mayor probabilidad de alteraciones en memoria, mientras que las anormalidades en sustancia blanca se relacionaban con déficits en atención y, específicamente, en velocidad de procesamiento de la información.

En la misma línea de investigación, Armstrong et al. ${ }^{(24,32,37-39)}$, fueron de los primeros autores que definieron el patrón de rendimiento de los procesos de memoria verbal y visual, a lo largo del tiempo en pacientes con gliomas de bajo grado. En relación a la memoria verbal, hallaron un declive en dicho dominio transcurridos tres meses de la finalización del tratamiento de RT, seguido de una mejoría en el rendimiento en estas pruebas hasta equiparar las puntuaciones previas a la aplicación del tratamiento a los doce meses después de haberlo finalizado. Los autores definieron los cambios en memoria verbal como efectos inmediatos de la RT, asociados a procesos de desmielinización $^{(32,39)}$, que se recuperaron de forma espontánea. En relación a la memoria visual, observaron déficits en este proceso cognitivo de forma previa a la aplicación del tratamiento de RT, seguidos de un patrón de recuperación a los doce meses que se mantuvo hasta el quinto año. A partir del sexto año de la aplicación de la RT, los autores identificaron un deterioro selectivo en memoria visual ${ }^{(24)}$. Para ambos procesos mnésicos, estos autores señalaron que las alteraciones se producen en las fases de recuperación de la información, sin identificarse alteraciones en aprendizaje, en la codificación y en funciones ejecutivas. Por ende, concluyeron que la mejoría observada en los cinco primeros años en el rendimiento en aprendizaje visual y en recuperación de esta información, se debe al beneficio de la RT sobre el control del tumor.

\section{b) Rendimiento neuropsicológico en función de la modalidad de tratamiento de radioterapia en pacientes con gliomas de bajo grado}

Dentro de los estudios que comparan el rendimiento neuropsicológico en función de la modalidad de tratamiento de RT aplicada, Gregor et al. ${ }^{(40)}$, realizaron una investigación transversal para valorar el rendimiento en áreas de atención, incluyendo velocidad de procesamiento de la información, de memoria visual, de organización visoespacial y el nivel de inteligencia, seis años después del tratamiento de RT. Estos autores compararon los resultados obtenidos en las pruebas neuropsicológicas entre pacientes que recibían RT holocraneal, con respecto a los que fueron tratados con RT focal. Los datos informaron de peores puntuaciones en organización visoespacial, velocidad de procesamiento de la información y memoria visual en aquellos pacientes que fueron tratados con RT holocraneal; además, estos pacientes presentaban una probabilidad siete veces mayor de alteraciones neuropsicológicas, concluyendo que el principal factor predictor de deterioro cognitivo era la RT holocraneal.

Los resultados aportados por estos últimos autores son similares a los referidos por el equipo de Surma-aho(26), que identificaron un mayor deterioro cognitivo en pacientes que recibieron RT holocraneal siete años después de la finalización de la misma, con respecto a aquellos que recibieron RT focal. Estos autores informaron de un peor rendimiento en tareas de memoria visual e inteligencia en los pacientes que recibían $\mathrm{RT}$, independientemente de la modalidad aplicada, con respecto a aqueIlos que no recibieron RT; siendo menor dicho funcionamiento en los pacientes que recibieron la modalidad holocraneal (68\% de la muestra total). Asimismo, señalaron que la presencia de leuco-encefalopatía, valorada mediante pruebas de neuroima- 


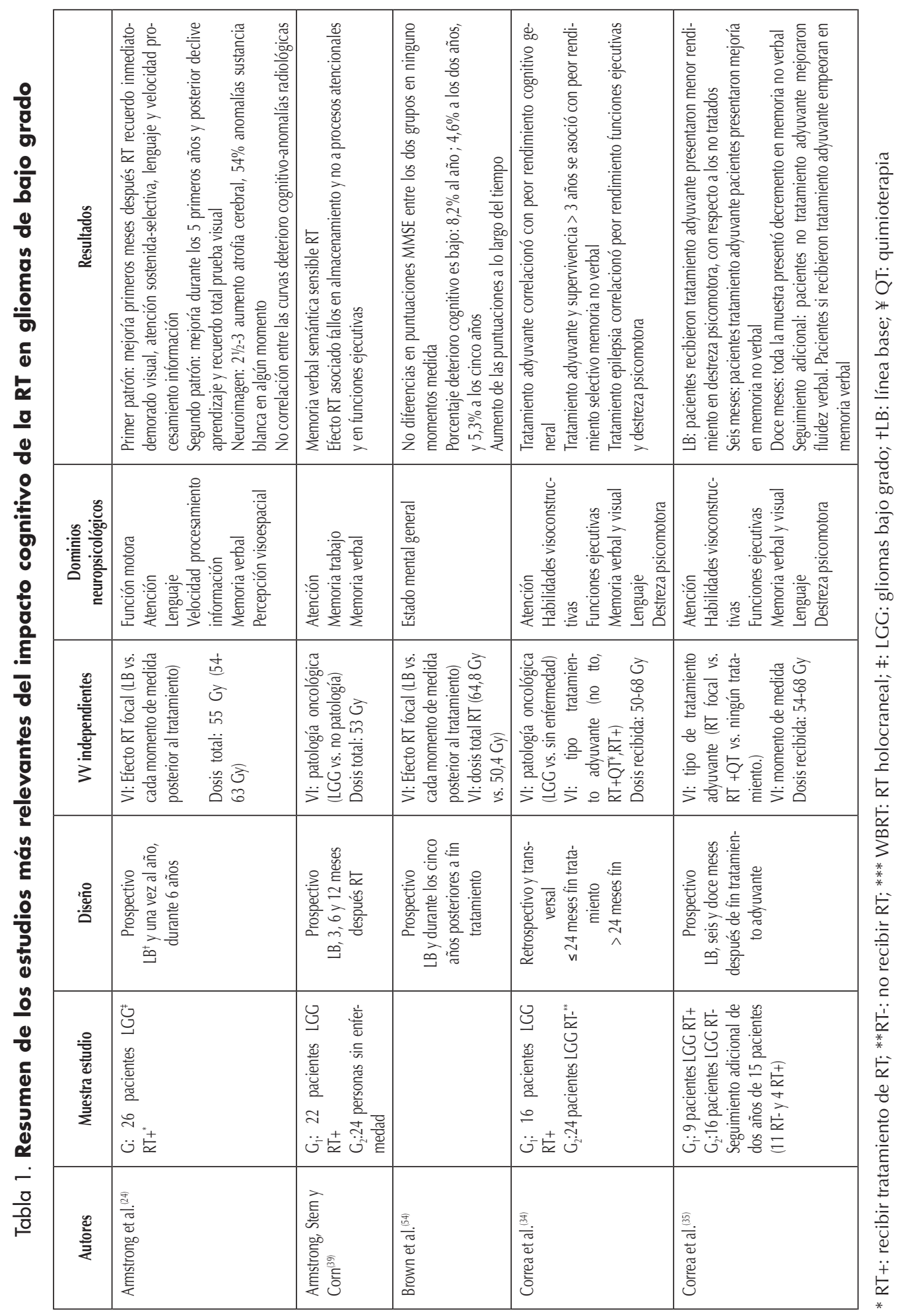




\begin{tabular}{|c|c|c|c|c|c|}
\hline 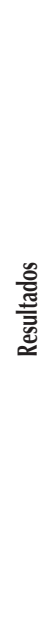 & 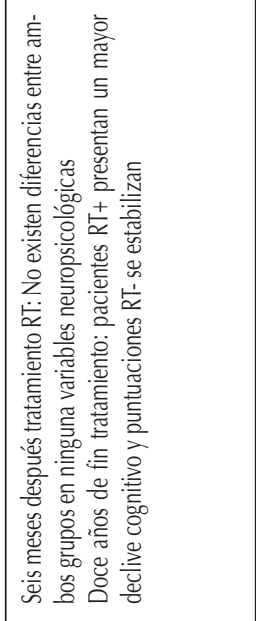 & 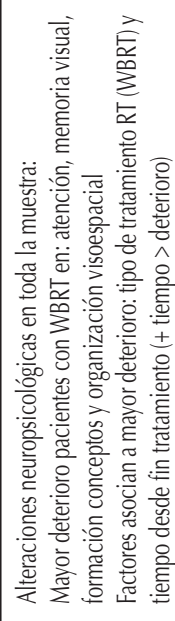 & 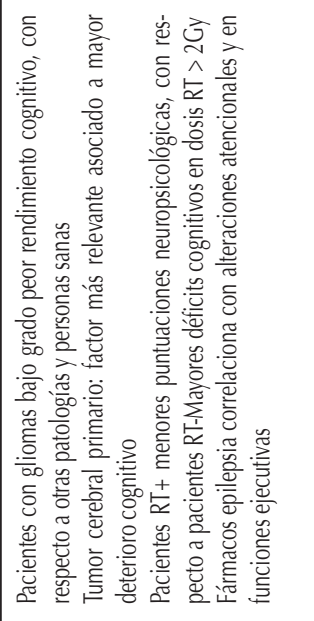 & 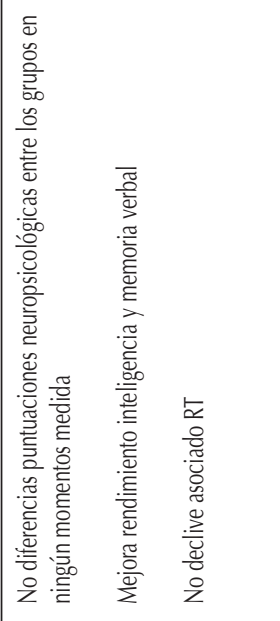 & 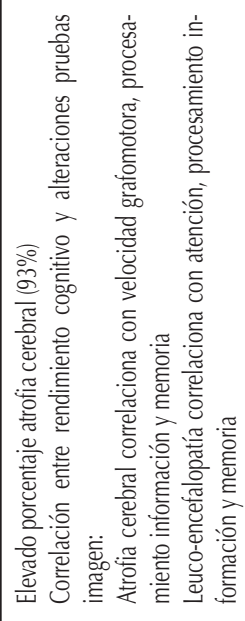 \\
\hline 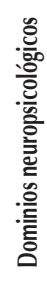 & 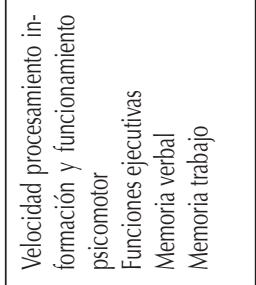 & 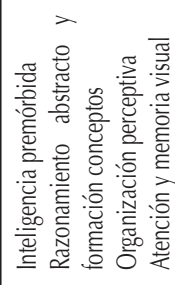 & 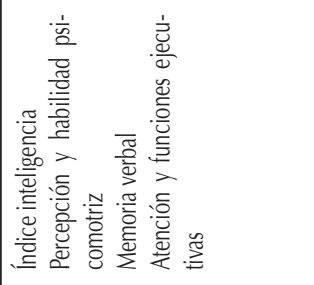 & 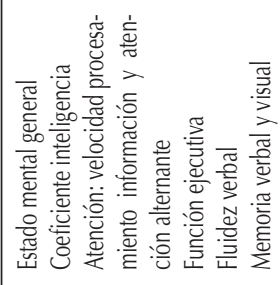 & 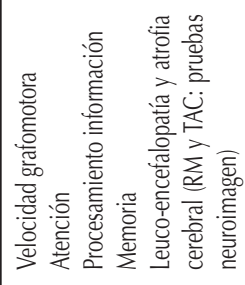 \\
\hline 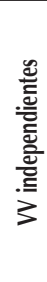 & 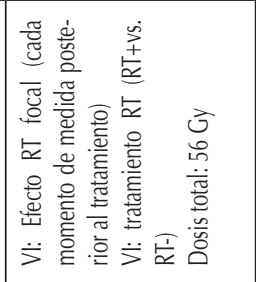 & 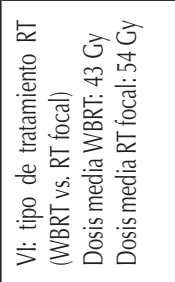 & 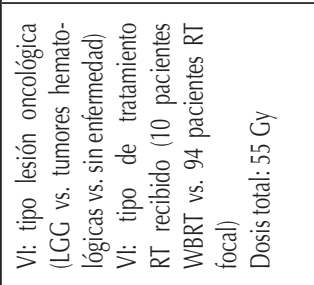 & 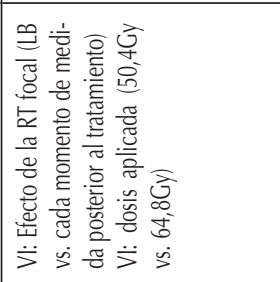 & 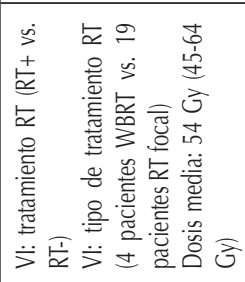 \\
\hline 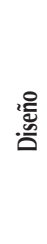 & 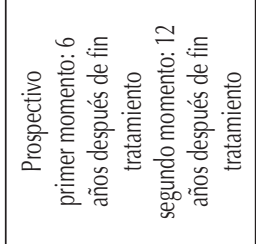 & 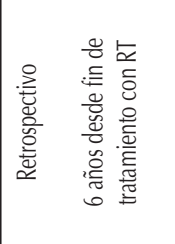 & 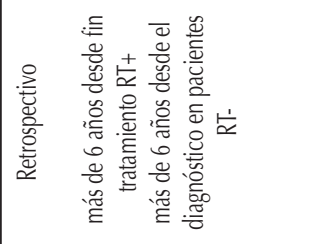 & 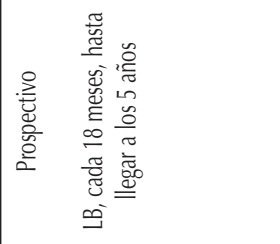 & 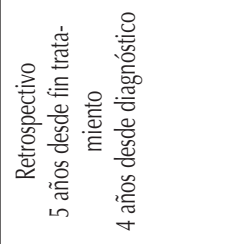 \\
\hline 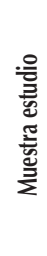 & 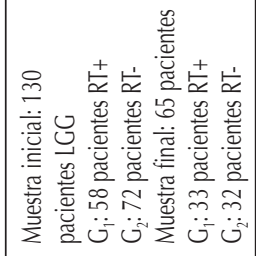 & 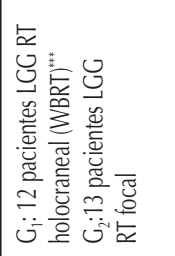 & 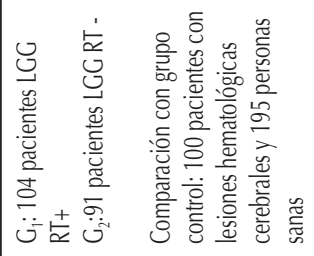 & 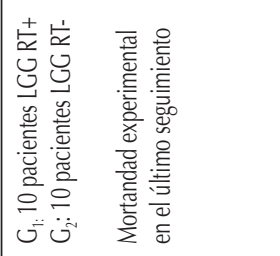 & 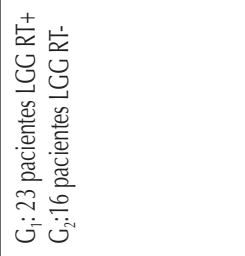 \\
\hline 递 & 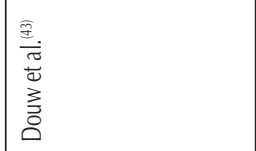 & 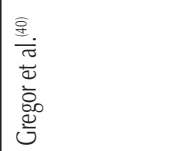 & 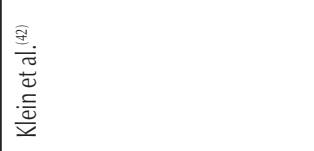 & 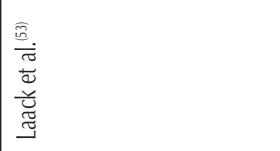 & 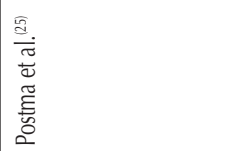 \\
\hline
\end{tabular}




\begin{tabular}{|c|c|c|c|c|}
\hline 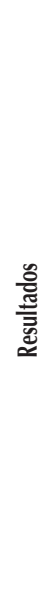 & 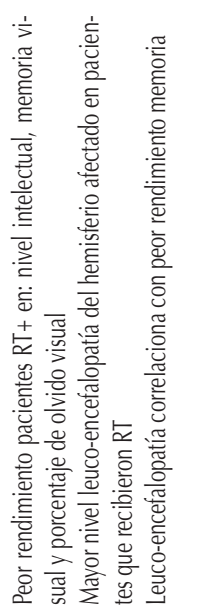 & 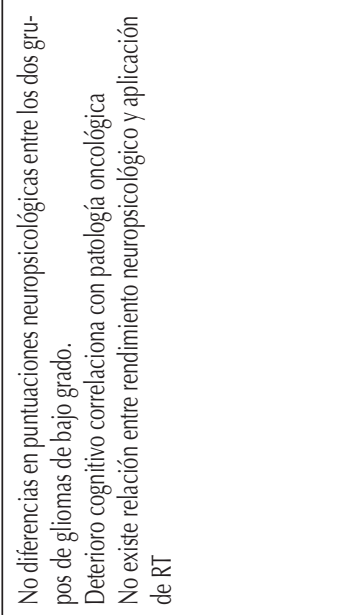 & 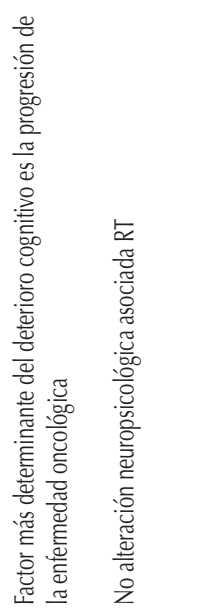 & 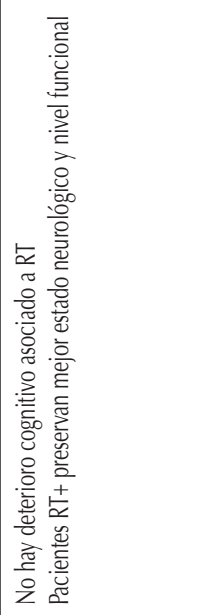 \\
\hline 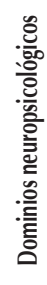 & 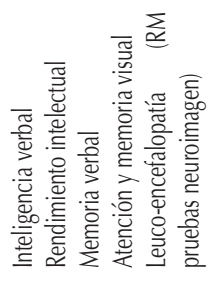 & 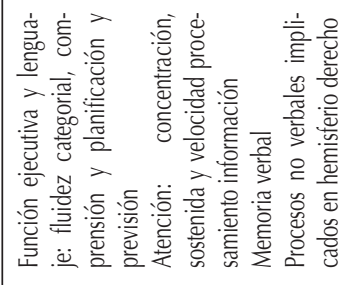 & 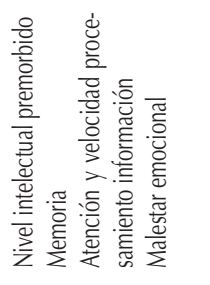 & 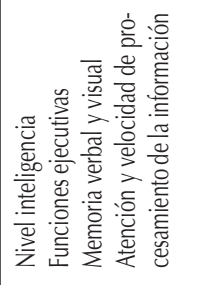 \\
\hline 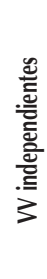 & 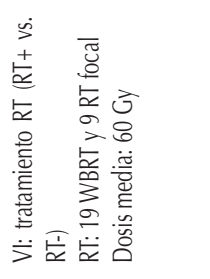 & 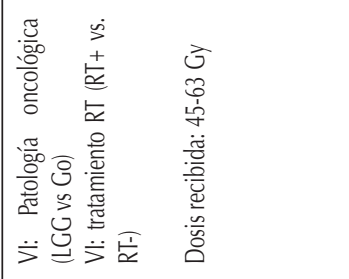 & 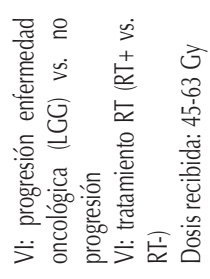 & 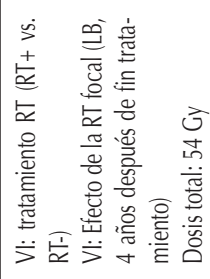 \\
\hline 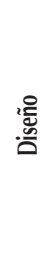 & 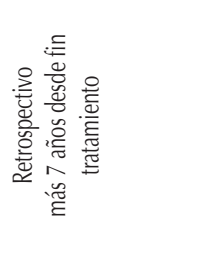 & 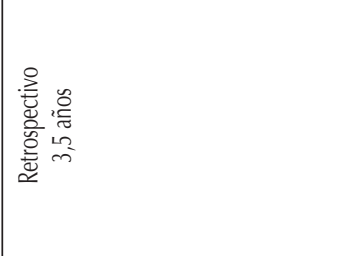 & 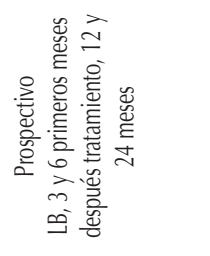 & 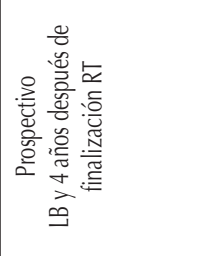 \\
\hline 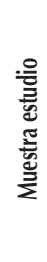 & 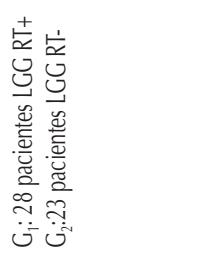 & 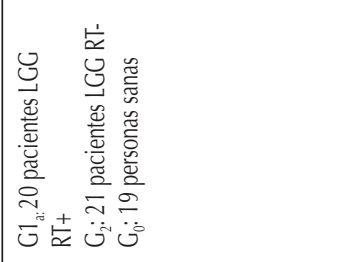 & 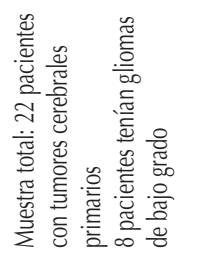 & 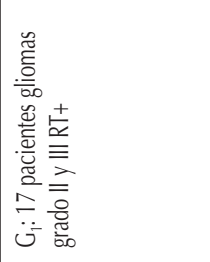 \\
\hline 递 & 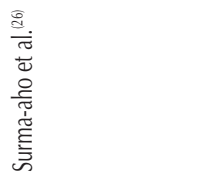 & 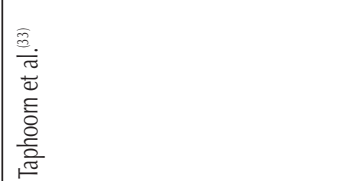 & 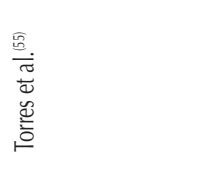 & 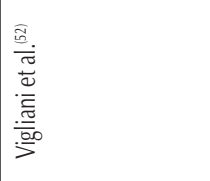 \\
\hline
\end{tabular}


gen, correlacionó de forma positiva con un pobre rendimiento en memoria visual.

Los resultados hallados en estos dos estudios, que asocian esta modalidad de tratamiento a fallos en diferentes dominios neuropsicológicos, corroboran los hallazgos de investigaciones en pacientes con metástasis cerebrales que se sometieron a RT holocraneal(41). En estas investigaciones se apunta a la sensibilidad demorada del hipocampo como la causa de las alteraciones en memoria visual, en atención, en organización visoespacial y en habilidades de formación de conceptos.

\section{c) Rendimiento neuropsicológico en función la dosis total y fraccionada de radiación recibida}

En último lugar, dos estudios documentan la asociación entre la dosis fraccionada de radiación recibida y la presencia de deterioro en algún dominio neuropsicológico. El equipo de Klein ${ }^{(42)}$ desarrolló un estudio con una gran muestra (195 pacientes con glioma de bajo grado), que incluyó los siguientes dominios neuropsicológicos: memoria, atención, percepción y funciones ejecutivas. Estos autores informaron que los pacientes que recibían dosis fraccionadas iguales o mayores a 2 Gy, presentaban mayor deterioro en memoria. No obstante, todos los pacientes con gliomas de bajo grado, hubieran o no recibido tratamiento adyuvante de RT, presentaron peor rendimiento en velocidad de procesamiento de la información y otras variables atencionales, memoria verbal y funciones ejecutivas, con respecto a pacientes con otras lesiones cerebrales y a personas sanas. De igual forma que Correa et al. ${ }^{(34)}$, estos investigadores señalaron que la aplicación de tratamiento anti-epileptógeno se asociaba con fallos en atención y funciones ejecutivas. Klein et al. ${ }^{(42)}$, concluyeron que la mejor variable predictora del deterioro cognitivo fue la lesión oncológica y que la RT, en dosis fraccionadas elevadas, generaba un efecto adicional al deterioro cognitivo a largo plazo.

Estos mismos autores han publicado recientemente un estudio ${ }^{(43)}$ con un largo seguimiento (12 años), recogiendo un amplio número de dominios neuropsicológicos. Los autores compararon las puntuaciones obtenidas en las diversas pruebas neuropsicológicas, a los seis y doce años de finalización del tratamiento, en pacientes que recibieron $\mathrm{RT}$ con respecto a aquellos que no recibieron dicho tratamiento. Entre los resultados más importantes, informaron que se mantenía el efecto de una elevada dosis de RT fraccionada (2Gy) en el funcionamiento mnésico, aportado en el estudio de Klein et al. ${ }^{(42)}$. Además, observaron en los pacientes que recibieron RT doce años después del mismo, un peor rendimiento en funciones ejecutivas, atención y velocidad de procesamiento de la información. Asimismo, indicaron la presencia de alteraciones clínicas en funciones ejecutivas, funcionamiento psicomotor, memoria de trabajo y atención, a los doce años en el grupo de pacientes que recibió RT.

En relación a la dosis total aplicada, diferentes autores ${ }^{(9,27,44)}$ han señalado que radiaciones iguales o superiores a 60 Gy se asocian a un mayor riesgo de presentar alteraciones neuropsicológicas. Así, la mayor parte de estudios en los que se observan alteraciones en la memoria visual $^{(22,34,35)}$ o en otras funciones neuropsicológicas como por ejemplo atención ${ }^{(25,43)}$, inteligencia general ${ }^{(26)}$, fluidez fonológica ${ }^{(35)}$, así como funciones ejecutivas $^{(43)}$, las dosis máximas aplicadas a los pacientes fueron superiores a 60 Gy. En este sentido, la EORTC ${ }^{(45)}$ advirtió que los pacientes con gliomas de bajo grado que recibieron dosis totales de 59,4 Gy referían menor funcionamiento cognitivo auto-referenciado, valorado mediante pruebas de calidad de vida específicas para tumores cerebrales. 


\section{d) Otros factores asociados al deterioro cognitivo de la radioterapia}

Además de los factores de la RT referidos en los apartados anteriores, que se recogen en la Tabla 2, en este punto se citan otras variables propias de esta técnica y otros factores del paciente que pueden influir en deterioro cognitivo radio-inducido en personas con gliomas de bajo grado. En relación a la variable tiempo, el transcurso desde la finalización del tratamiento y la cronicidad de la enfermedad podrían potenciar la presencia de alteraciones neuropsicológicas en la población estudiada. El dato es corroborado por los estudios de Correa et al. ${ }^{(34,35)}$, y Armstrong et al. ${ }^{(24)}$, en los que se evidencia que entre el tercer y quinto año de la finalización del tratamiento los pacientes comenzaban a presentar un deterioro selectivo en memoria visual. Además, en otra investigación (30) se ha señalado que los pacientes con mayor tiempo de supervivencia presentaban una mayor probabilidad de este tipo de déficits, a pesar de no recibir tratamientos adyuvantes.
Respecto a las bases anatómicas relacionadas con estos factores de la RT, Sirkkisian ${ }^{(46)}$ señaló que la aplicación de este tratamiento se asocia con un aumento de la regulación del factor de crecimiento endotelial vascular, debido a hipoxia cerebral en la sustancia blanca, de forma exclusiva. Este autor señala que la extensión del daño es dependiente de la dosis de RT recibida, pudiendo explicar la presencia de deterioro cognitivo en pacientes que reciben dosis elevadas totales y fraccionadas. Asimismo, informa que se produce un deterioro de la neurogénesis del hipocampo posterior a la RT, debido a procesos de inflamación que se podrían asociar a fallos en atención y memoria visual.

Por último, se enumeran los factores del paciente que se asocian con el desarrollo de deterioro cognitivo. La variable edad es el factor más determinante en la aparición de deterioro cognitivo en población general y en los pacientes con gliomas, tal y como se recoge en un estudio reciente ${ }^{(19)}$. Además, la presencia de una enfermedad neurológica previa al diagnóstico de glioma de bajo grado, como

\section{Tabla 2. Factores asociados con el deterioro cognitivo radio-inducido}

- Factores de la radiación:

1. Dosis total aplicada

2. Modalidad del tratamiento RT (holocraneal vs. focal)

3. Dosis fraccionada aplicada

4. Tiempo transcurrido desde diagnóstico y fin del tratamiento

5. Duración total de la radioterapia

6. Volumen total de tejido cerebral irradiado

- Factores del paciente:

1. Edad

2. Predisposición genética

3. Enfermedades neurológicas previas

4. Antecedentes enfermedad sistémica

5. Tratamiento QT concomitante 
por ejemplo la esclerosis múltiple, puede influir de forma negativa en el rendimiento neuropsicológico del paciente ${ }^{(47)}$. En la misma línea, ciertas enfermedades sistémicas, tales como la hipertensión arterial o la diabetes, pueden favorecer la existencia de un deterioro cognitivo ligero $^{(48,49)}$. Tal y como indican, Uribe y González ${ }^{(50)}$ otro factor predictor del daño sobre el SNC modulado por la RT es presentar antecedentes de síndromes de inestabilidad genética (ataxia telangiectásica, Von Recklinghausen). Un último factor a considerar en el estudio del rendimiento neuropsicológico en estos pacientes, es la administración de tratamientos de quimioterapia de forma concomitante a la RT. En este sentido, Taphoorn y Klein ${ }^{(9)}$ recogen el impacto de la Carmustina y del Cisplatino a nivel cognitivo, a pesar de ser fármacos poco utilizados en pacientes con gliomas de bajo grado. Estos mismos autores señalaron que la utilización de altas dosis de Vincristina se asocian con leucoencefalopatía y con deterioro cognitivo en estos pacientes e informan de la necesidad de realizar estudios prospectivos que valoren el impacto neuropsicológico de las nuevas sustancias de quimioterapia, aplicadas en pacientes con gliomas como puede ser el caso de la Temozolamida.

\section{DATOS EN CONTRA DE LA EXISTENCIA DE DETERIORO COGNITIVO ASOCIADO A LA RT}

El número de investigaciones que demuestra la ausencia de deterioro neuropsicológico asociado a la RT es menor, únicamente se han hallado seis estudios, realizados la mayor parte en la década de los noventa. Este hecho dificulta la generalización de los resultados en la actualidad, ya que el avance en las técnicas de tratamiento conlleva una mayor seguridad de los tratamientos y una disminución de los efectos neurotóxicos.
Taphoorn et al. ${ }^{(33)}$, fueron unos de los primeros investigadores que valoraron el daño cognitivo asociado a la RT, tres años y medio después de la finalización del tratamiento, a partir de una investigación de carácter transversal; los autores señalaron que la patología oncológica fue la variable encargada de explicar los fallos a nivel cognitivo y el bajo estado de ánimo de los pacientes con gliomas de bajo grado, y no el tratamiento de RT.

En la misma línea que los autores anteriores, Hammack et al. ${ }^{(51)}$, valoraron diferentes dominios neuropsicológicos (nivel intelectual, aprendizaje y memoria) en una muestra formada por 19 pacientes con gliomas de bajo grado, en dos momentos de medida (previamente a la aplicación de la RT y cinco años después de la misma). Estos autores concluyeron que no existía déficits en los dominios valorados cinco años después de la aplicación del tratamiento, siendo estos datos similares a los aportados por Armstrong et al. (24) que informaron de ausencia de deterioro cognitivos a los cinco años de finalizar la RT, aunque no por ello se debe descartar la presencia de un deterioro cognitivo posterior a este momento.

En otros dos estudios de carácter longitudinal no se evidenció deterioro cognitivo asociado a la RT en pacientes con gliomas de bajo grado ${ }^{(52,53)}$, señalándose en ambos una mejoría neurológica o neuropsicológica posterior a la RT en estos pacientes. Así, Vigliani et al. ${ }^{(52)}$, compararon el rendimiento neuropsicológico antes del comienzo de la RT y cuatro años después del tratamiento, en diferentes dominios (funciones ejecutivas, memoria verbal y visual, nivel inteligencia, atención y velocidad de procesamiento de la información); así como, compararon el rendimiento en estos dominios entre pacientes que recibieron RT, con respecto a aquellos que no necesitaron este tratamiento. Además de no observar la presencia de deterioro cognitivo, a 
nivel global en los pacientes que recibieron la RT; los autores informaron de una mayor duración de un estado neurológico preservado y un nivel funcional adecuado en el grupo de pacientes que recibieron $\mathrm{RT}$, con respecto a los pacientes que no fueron tratados con dicho tratamiento. Laack et al. ${ }^{(53)}$, valoraron un amplio número de funciones cognitivas en pacientes que recibieron $\mathrm{RT}$, antes de la aplicación de la misma y cada 18 meses después de la RT, hasta llegar a un seguimiento máximo de cinco años. Estos autores informaron que todos los pacientes tratados con gliomas de bajo grado presentaban un menor rendimiento neuropsicológico en todas las áreas, con respecto a la población general. Además, no observaron un deterioro cognitivo asociado a la $\mathrm{RT}$, informando de una mejoría 18 meses después de la misma en medidas de inteligencia y memoria verbal para los pacientes que habían recibido este tratamiento, con respecto a aquellos que no lo hicieron.

Brown et al. ${ }^{(54)}$, son unos de los pocos autores que han utilizado el Mini Mental State Examination (MMSE) para determinar el impacto cognitivo de la RT en pacientes con gliomas de bajo grado. Estos aplicaron esta prueba antes de la realización del tratamiento y en diferentes momentos después del mismo (cada 4 meses durante los 2 primeros años, cada 6 meses los 3 años siguientes y 1 vez al año posteriormente), determinando como cambio significativo en el rendimiento cognitivo si se producía un aumento o disminución de tres puntos. Los autores señalaron que no se produjo deterioro cognitivo a lo largo del tiempo en la muestra estudiada, así como no se observaron diferencias en las puntuaciones, en ningún momento de medida, entre los pacientes que recibieron una dosis de radiación total elevada (64,8Gy), con respecto a aquellos que recibieron dosis menores (50,4Gy). Estos autores concluyeron, que los pacientes podrían haber experimentado alteraciones en memoria, funciones ejecutivas o en otros dominios neuropsicológicos que no pudieron ser identificadas por el test aplicado, ya que este instrumento carece de la sensibilidad y especificidad necesaria para identificar los cambios cognitivos asociados a los tratamientos oncológicos.

En último lugar, el estudio realizado por Torres et al. ${ }^{(55)}$, fue uno de los pioneros en determinar el impacto neuropsicológico de las técnicas de RT modernas (modern highly conformal fractionated partial) en pacientes con gliomas de bajo grado. Para ello, realizaron una investigación longitudinal prospectiva con medidas repetidas (línea base y hasta dos años después de la RT) y aplicaron una batería neuropsicológica que recogía información de inteligencia, memoria y atención. Los autores determinaron que el deterioro cognitivo observado en dominios de atención y memoria, se asociaba a la progresión de la enfermedad oncológica, sin evidenciarse relación con el tratamiento de RT aplicado.

\section{LIMITACIONES METODOLÓGICAS DE LOS ESTUDIOS DEL IMPACTO COGNITIVO DE LA RADIOTERAPIA EN PACIENTES CON GLIOMAS DE BAJO GRADO}

Es necesario considerar una serie de aspectos metodológicos, que pueden influir en la variabilidad de los resultados expuestos en el presente artículo, que abarcan desde el diseño empleado en el estudio hasta la definición de las variables neuropsicológicas estudiadas.

\section{a) Diseño}

Los diseños de carácter transversal permiten, únicamente, valorar la prevalencia del deterioro cognitivo en un momento determinado, por lo que no se puede 
observar el patrón de rendimiento en las variables neuropsicológicas a lo largo del tiempo, ni sus posibles fluctuaciones. Por ello, los resultados obtenidos en estos estudios no serán los más adecuados para generalizar los datos obtenidos, ni aportar las conclusiones sobre el impacto de esta técnica a nivel neuropsicológico a lo largo del tiempo, salvo en el momento de medida al que se ciñan.

Además, en los estudios de naturaleza transversal revisados se evidencia una falta de consideración en los análisis estadísticos del tiempo transcurrido desde el fin de la RT, igualando los resultados de pacientes que finalizaron el tratamiento un año antes de la valoración neuropsicológica, con respecto a aquellos que lo hicieron hasta ocho años después.

Una última limitación de este tipo de diseño, es la ausencia de información del estado cognitivo previo a la aplicación de la RT, variable determinante en el daño que pudiera generar esta técnica en procesos neuropsicológicos y en el pronóstico de esta población, como se ha señalado previamente $^{(8)}$.

Estas deficiencias son subsanadas en los estudios longitudinales que sí permiten determinar el estado neuropsicológico previo a la RT y su patrón de fluctuación en el tiempo, pero la falta de acuerdo en los momentos de medida (algunos autores valoran cada 3 o 6 meses mientras que otros los hacen cada 18 meses); así como, el escaso seguimiento que se obtiene en algunos estudios (tres meses o doce meses) dificultan el esclarecimiento de los resultados.

El problema más importante que se deriva de los estudios de carácter longitudinal, es la falta de consideración, en algunos de ellos, de los diversos efectos de la RT (asociados a distintas etiologías), que van a provocar alteraciones cognitivas diferentes. Así, no debería compararse el daño que se produce en los primeros meses posteriores a la RT por procesos de desmielinización ${ }^{(34)}$, con aquellos que se producen años después, que tienen un carácter irreversible y un mayor impacto en los procesos neuropsicológicos ${ }^{(24,26,43)}$.

En relación a este último tipo de deterioro cognitivo, se han encontrado únicamente dos estudios con seguimientos mayores de seis años después de la RT, que informan de deterioro selectivo en memoria visual a partir del quinto año y se mantiene doce años después. Por ello y por la larga supervivencia de estos pacientes, sería aconsejable que futuros investigadores diseñaran estudios con medidas repetidas y largos seguimientos en el tiempo, que ayuden a esclarecer el papel de la RT en el rendimiento neuropsicológico de esta población.

\section{b) Instrumentos de medida}

En la actualidad existen un importante número de cuestionarios neuropsicológicos con buenas propiedades psicométricas y con baremación, principalmente en población norteamericana. Este hecho permite tener una amplia variabilidad para valorar una función cognitiva o proceso neuropsicológico específico, tal y como se recoge en la Tabla 3. No obstante, se considera que esta variabilidad conlleva un importante inconveniente, ya que la utilización de diferentes instrumentos para medir el mismo dominio puede dar lugar a resultados contradictorios. Así, la aplicación de un determinado cuestionario puede conllevar que se recoja información de un proceso cognitivo específico del dominio neuropsicológico evaluado $y$, a su vez, dicho proceso puede que no estar recogido en otros test neuropsicológicos que valoran el mismo dominio.

Por otra parte, pocos autores han incluido todos los dominios cognitivos en las valoraciones neuropsicológicas, probablemente para evitar el efecto fatiga en los 


\section{Tabla 3. Instrumentos de valoración neuropsicológica aplicados en los estudios revisados}

\begin{tabular}{|c|c|}
\hline $\begin{array}{c}\text { Dominios } \\
\text { neuropsicológicos }\end{array}$ & Instrumentos de medida \\
\hline Funciones ejecutivas & $\begin{array}{l}\text { - Test de Colores y Palabras Stroop } \\
\text { - Tarea de fluidez fonológica subescala del COWAT } \\
\text { - Concept-shifting test } \\
\text { - Span dígitos y palabras WAIS-IIII }{ }^{(59)} \\
\text { - Subescalas de Matrices de la escala WISC } \\
\text { - Wisconsin Card Sorting Test }{ }^{(61)} \\
\text { - Tarea de memoria de trabajo } \\
\end{array}$ \\
\hline Lenguaje & $\begin{array}{l}\text { - Tarea de fluidez categorial subescala del COWAT } \\
\text { - Sentence Repetition Test }{ }^{(62)} \\
\text { - Controlled Oral Word Association Test (COWAT) }{ }^{(57)}\end{array}$ \\
\hline Atención: & $\begin{array}{l}\text { - Test d2 }{ }^{(63)} \\
\text { - Trail Making Test }(\mathrm{A}-\mathrm{B})^{(64)} \\
\text { - Subtest Dígitos y Símbolos WAIS-III(59) } \\
\text { - Test de Colores y Palabras Stroopp }{ }^{(56)} \\
\text { - Auditory Selective Attention Test }{ }^{(65-66)} \\
\text { - Graded Naming Test }{ }^{(67)} \\
\text { - Continous Performance Test }{ }^{(68)} \text { Bells Test }^{(69)}\end{array}$ \\
\hline Memoria verbal & $\begin{array}{l}\text { - Test Auditivo-Verbal aprendizaje Rey }{ }^{(70)} \\
\text { - Hopkins Verbal Learning Test }{ }^{(71)} \\
\text { - Selective Reminding } \\
\text { - Recall memory test }{ }^{(36)} \\
\text { - Tarea de recuerdo de semejanzas WAIS-IIII } \\
\text { - Digit span test } \\
\text { - Word span test }{ }^{(73)}\end{array}$ \\
\hline Memoria visual & $\begin{array}{l}\text { - Test Retención visual Benton } \\
\text { - Test de Reconocimiento facial Benton } \\
\text { - Test de la Figura Compleja de Rey } \\
\text { - Visual association test } \\
\text { - 10/26 Spatial Recall Test } \\
\end{array}$ \\
\hline $\begin{array}{l}\text { Organización visoespa- } \\
\text { cial y perceptiva }\end{array}$ & $\begin{array}{l}\text { - Subescala de Diseño cubos del WAIS III(59) } \\
\text { - Subescala de Ensamblaje objetos del WAIS IIII }{ }^{(59)} \\
\text { - Test de la Figura Compleja Rey }{ }^{(76)} \\
\text { - Judgment of line orientation test }{ }^{(79)} \\
\text { - Line bisection test }{ }^{(80)} \\
\text { - Visospatial judgment test }{ }^{(81)} \\
\text { - Road map test }{ }^{(82)} \\
\text { - Visual pursuits test }{ }^{(36)} \\
\text { - Visual Object and Space Perception Battery }{ }^{(83)} \\
\text { - Incomplete letters }{ }^{(35)} \\
\text { - Hayling sentence completion test }{ }^{(84)} \\
\text { - Brixton spatial anticipation test }{ }^{(84)}\end{array}$ \\
\hline
\end{tabular}




\begin{tabular}{|c|c|}
\hline $\begin{array}{c}\text { Dominios } \\
\text { neuropsicológicos }\end{array}$ & Instrumentos de medida \\
\hline $\begin{array}{l}\text { Inteligencia } \\
\text { premorbida }\end{array}$ & $\begin{array}{l}\text { - Span dígitos y semejanzas del WAIS IIII59) } \\
\text { - Diseño bloques y Dígitos-Símbolos del WAIS IIII(59) } \\
\text { - Dutch adult Reading Test }{ }^{\left({ }^{(5)}\right)} \\
\text { - National Adult Reading Test }{ }^{(86)} \\
\text { - Wechsler Adult Intelligence Scale-Third Edition (WAIS-III) }{ }^{(59)} \\
\text { - Subescala de Semejanzas del WAIS IIII } \\
\text { - Subescala de Comprensión el WAIS-III(59)} \\
\text { - Shipley Institute of Living Scale }{ }^{(87)}\end{array}$ \\
\hline $\begin{array}{c}\text { Velocidad grafomotora } \\
\text { y habilidad } \\
\text { psicomotriz }\end{array}$ & $\begin{array}{l}\text { - Letter-digit substitution test }{ }^{(88)} \\
\text { - Finger zapping test }{ }^{(89)} \\
\text { - Concept Shifting Test } \\
\text { - Paced Auditory Serial Addition Test }{ }^{(90)} \\
\text { - Pegboard Test }{ }^{(91)}\end{array}$ \\
\hline Estado mental general & - Mini-Mental State Examination (MMSE) ${ }^{(92)}$ \\
\hline
\end{tabular}

pacientes, siendo más frecuentemente valorados los dominios atencionales y mnésiCos, así como el nivel intelectual| ${ }^{25,26,42,43,55)}$. Debido a que actualmente está demostrada la sensibilidad del hipocampo y del sistema límbico a la RT, específicamente en la modalidad holocraneal(41,93), en futuros estudios sería recomendable considerar en las valoraciones neuropsicológicas habilidades asociadas a dichas estructuras, como por ejemplo estrategias de planificación y organización, flexibilidad mental, interferencia, solución de problemas y funcionamiento psicomotor entre otras.

En último lugar, se han indicado una serie de características que deben recoger los cuestionarios aplicados, para aumentar la eficacia de las valoraciones y la representatividad de los resultados obtenidos. Diferentes autores ${ }^{(9,29)}$ han incidido en la necesidad de seleccionar cuestionarios de valoración neuropsicológica con una óptima fiabilidad test-retest, que presenten formas alternativas para evitar el efecto de la práctica y con una adecuada sensibilidad a los efectos neurotóxicos de los tratamientos adyuvantes. Para ello, Taphoorn y $\operatorname{Klein}^{(9)}$ han propuesto una batería de instrumentos neuropsicológicos que incluyen: a) dominios de velocidad de procesamiento de la información, percepción; b) memoria verbal, visual y memoria de trabajo; c) atención y funciones ejecutivas, y d) funcionamiento intelectual premórbido.

\section{c) Muestra}

Otra cuestión metodológica a considerar, es el tamaño de la muestra de los estudios revisados, ya que a excepción de las investigaciones realizadas por el equipo de Klein y Douw ${ }^{(42,43)}$, el número de participantes que recibían tratamiento de RT no ha superado los 30 sujetos y en los estudios prospectivos se observa una importante mortandad experimental, como por ejemplo ocurre en el estudio realizado por Armstrong et al. ${ }^{(24)}$, y Laack et al. ${ }^{(53)}$. Este hecho se corresponde con la realidad clínica de la población estudiada, ya que la prevalencia de estas lesiones es baja como se ha apuntado anteriormente, y además existe una dificultad en los seguimientos de estos pacientes, debido al em- 
peoramiento de su estado neurológico y a la progresión de la enfermedad a mayores grados de malignidad. Estos hechos repercuten en la obtención de información de aquellos pacientes con peor estado cognitivo que tiene un valioso interés científico.

Se considera importante señalar que las investigaciones en las que no se evidenciaba una relación entre deterioro cognitivo y aplicación de RT, tenían tamaños muestrales que no superaban los 20 pacientes con gliomas de bajo grado, lo que dificulta la generalización de los resultados. Además, en dos de éstas la población estudiada incluía lesiones oncológicas con diferentes grados de malignidad (grado II y grado III) $)^{(52)}$ y tumores de diferente histología a la estirpe glial(55), aspectos que podrían estar interfiriendo en los resultados aportados. Así, los gliomas de grado III al presentar una mayor naturaleza inflintrante y un mayor grado de malignidad, pueden afectar a un mayor número de estructuras cerebrales, ocasionando una mayor afectación neuropsicológica. Asimismo, en este tipo de lesiones la aplicación de RT siempre va acompañada de un tratamiento de quimioterapia concomitante, pudiendo aumentar los efectos secundarios de neurotoxicidad en el SNC e implicar un mayor número de alteraciones neuropsicológicas. Por otra parte, las lesiones de naturaleza histológica diferente a la estirpe glial, pueden asociarse con un menor impacto neurológico y cognitivo, debido a que estas lesiones tienen un mejor pronóstico, presentan menos complicaciones clínicas y su localización es extra-axial, por lo que no se generaría un daño directo en las estructuras corticosubcorticales implicadas en los procesos neuropsicológicos valorados.

Una última consideración de la muestra, es la aleatorización de los pacientes a los grupos de tratamiento (recibir o no RT). Los pacientes con un estado neurológico deficitario o en los que no se pudo realizar una exéresis completa del tumor fueron los candidatos para recibir el tratamiento de RT, pudiendo presentar un peor rendimiento neuropsicológico premórbido ${ }^{(24,42) .}$ Este hecho implica un sesgo en los resultados, al atribuirse a la RT un impacto sobre los procesos neuropsicológicos que podría deberse a la enfermedad oncológica en sí misma $^{(30,42,43)}$.

\section{CONCLUSIONES}

Uno de los resultados más relevantes que se derivan de estos estudios, es que los pacientes con gliomas de bajo grado presentan un rendimiento neuropsicológico peor que la población general, de forma previa a la aplicación de tratamientos adyuvantes. A pesar de que estos pacientes se han sometido a resección quirúrgica o biopsia del tumor, parece que la lesión oncológica en sí misma es la principal causa del deterioro cognitivo ${ }^{(9,27,30,31)}$. En esta línea, se han identificado una serie de factores, adicionales a la RT, que pueden precipitar o agravar las alteraciones cognitivas descritas en esta población, como por ejemplo el uso de fármacos antiepileptógenos, el tratamiento de corticoides, la cirugía, los tratamientos de QT adyuvantes, la presencia de trastornos de ansiedad y/o depresión, así como la combinación de todos ellos ${ }^{(30,31)}$.

En este campo, el impacto cognitivo de la RT ha sido una de las áreas que mayor interés ha suscitado por su implicación en la práctica clínica, generándose en estas dos últimas décadas un aumento en el número de investigaciones, que pretenden determinar el papel de esta técnica en el rendimiento neuropsicológico de los pacientes con gliomas de bajo grado. A pesar de la divergencia de los resultados en este área, en la mayor parte de estudios se ha informado de la relación entre deterioro cognitivo y RT, desde los primeros meses de la finalización del tratamiento hasta doce años después del mismo. Dentro de 
los dominios neuropsicológicos valorados, parece existir consenso en que la memoria visual es el más sensible a la RT, aunque se ha observado también un deterioro en funciones ejecutivas, funcionamiento psicomotor, velocidad de procesamiento de la información y otros dominios atencionales.

Otros de los resultados más importantes que se derivan de los estudios revisados, es la presencia de una fluctuación en el patrón de rendimiento neuropsicológico, específicamente de la memoria visual, que podría explicarse por las diferentes etiologías de los efectos neurológicos de la RT. En este sentido, se informa de un patrón de declive en este dominio entre los tres y seis meses después de su aplicación, asociado a procesos de desmielinización, que se acompaña de una mejoría en la función que puede mantenerse desde este momento hasta los 3 o 5 años del fin de RT. Posteriormente, se produce un nuevo deterioro en memoria visual que puede presentarse a partir del tercer año de la finalización de la RT, asociado a atrofia cerebral y leuco-encefalopatía de carácter irreversible.

En relación a los mecanismos fisiopatológicos de la RT, se considera que la destrucción o alteración de la sustancia blanca, así como la atrofia cerebral son las responsables del deterioro cognitivo, aunque no están esclarecidos los procesos por los cuáles se producen. La tendencia actual señala que el daño en las neural stem cell es uno de los principales responsables de la sensibilidad del hipocampo y del sistema límbico a la RT, generalmente cuando se aplica la modalidad holocraneal. Estas células localizadas en el cuerno de Ammón y la zona subgranular del hipocampo, son las encargadas de reparar el daño cerebral que se produce por factores externos, como puede ser la RT. Así, estudios recientes ${ }^{(93)}$ en pacientes con metástasis cerebrales han revelado una reducción en su proliferación entre las 12 y las 48 horas posteriores a la aplicación de la RT, pudiendo afectar de forma negativa a la sustancia blanca de estructuras cerebrales próximas.

En relación al papel de la sustancia blanca a nivel cognitivo, hasta hace pocos años se asociaba únicamente con la velocidad de procesamiento, surgiendo en la actualidad un cuerpo de conocimiento que pone de manifiesto la implicación de dicha sustancia en el funcionamiento de diferentes procesos cognitivos ${ }^{(94)}$. El mecanismo subyacente parece ser la noción de redes neurales distribuidas que conectan diferentes estructuras cerebrales, causando una desconexión entre las mismas si se produce una alteración o daño en la sustancia blanca. Tirapu-Ustárroz et al. ${ }^{(94)}$, ponen de manifiesto que la afectación de ésta se relaciona con diversas alteraciones del lenguaje, como por ejemplo diferentes tipos de afasia, afemia, dislexia o alexia sin agrafía. Estos autores también informan que en diversos estudios se asocian los daños en sustancia blanca, principalmente del hemisferio derecho, con déficits en todos los niveles atencionales. Además, señalan que las vías que sustentan los procesos visoespaciales incluyen la sustancia blanca de diferentes estructuras de áreas parieto-temporales derechas. En relación a la memoria, parece que actualmente un amplio número de investigaciones defiende la correlación entre la integridad de los haces de sustancia blanca y las capacidades de la memoria, asociándose la alteración de la sustancia blanca de diferentes estructuras con el déficit en un tipo de memoria específico. Además, se ha señalado que las funciones mnésicas parecen ser las más resistente a la leucoaraiosis pero cuando se alteran afectan principalmente a procesos memoria visual, información congruente con la aportada en los estudios revisados. En último lugar, la relación entre funciones ejecutivas y sustancia blan- 
ca de estructuras frontales parece ser la menos estudiada hasta la fecha, aunque sí existen datos que manifiestan el papel desarrollado por dicha sustancia en tareas de flexibilidad cognitiva e inhibición ${ }^{(94)}$.

Entre los factores de la RT que se asocian a un mayor deterioro cognitivo, el volumen de tejido cerebral irradiado y la dosis de radiación total y fraccionada parecen tener un papel determinante en el desarrollo y severidad de este deterioro. Se estima que dosis totales superiores a 60 Gy y dosis fraccionadas iguales o superiores a 2 Gy tienen una mayor probabilidad de deterioro neuropsicológico, principalmente de memoria visual y funcionamiento psicomotor. No obstante, estos datos surgen de estudios que han utilizado técnicas de RT convencionales, sin considerar el avance en las estrategias de tratamiento de estos últimos años, lo que implica que los resultados aportados por estos deben ser interpretados de forma cauta. Así, las mejoras en los protocolos de tratamiento incluyen nuevos mecanismos de inmovilización del paciente, una optimización de las técnicas de neuroimagen y un avance tecnológico en las técnicas terapéuticas, que incluyen RT tridimensional conformada, RT de intensidad modulada (IMRT) y RT guidada por imagen (IGRT) ${ }^{(95)}$. Todo ello, implica una menor radiación en el tejido cerebral sano, un aumento en la seguridad de los tratamientos y una disminución de los efectos tóxicos de la RT en las funciones neurológicas y cognitivas, haciéndose necesario la realización de nuevas investigaciones que permitan aclarar el papel de estas nuevas técnicas en el rendimiento neuropsicológico de los pacientes.

En el presente artículo se han repasado los aspectos metodológicos que pueden estar influyendo en la divergencia de los resultados hallados, incluyendo desde las características del diseño hasta los instrumentos de valoración neuropsicológicas utilizados. Se ha señalado la conveniencia de realizar investigaciones longitudinales prospectivas, que permitan determinar el estado neuropsicológico previo a la aplicación de los tratamientos adyuvantes, así como el patrón de funcionamiento a lo largo del tiempo. Además, se aconseja seleccionar muestras lo más homogéneas posible, en relación a la patología oncológica y al tratamiento de RT para facilitar la comparación de los resultados obtenidos. En relación a la valoración neuropsicológica, se considera necesario utilizar instrumentos con baremos de población general, si fuera posible, y con óptimas cualidades psicométricas, que presenten una sensibilidad adecuada para medir los cambios asociados a los efectos neurocognitivos de los tratamientos oncológicos.

Un último aspecto a considerar, es que la correcta interpretación de los resultados neuropsicológicos es decisiva para realizar un diagnóstico adecuado, determinar la afectación de ciertos procesos cognitivos y las habilidades preservadas ${ }^{(96)}$. Para ello, no se debe estimar únicamente sí la puntuación numérica obtenida en una prueba, se encuentra por encima o por debajo de los valores normativos ${ }^{(96)}$. Es necesario conocer los modelos teóricos de los dominios neuropsicológicos, tener en cuenta los procesos cognitivos implicados en cada uno de ellos ${ }^{(34,35,97)}$, así como considerar las variables socioculturales, neurológicas y médicas que pueden estar interfiriendo en dicho rendimiento.

\section{REFERENCIAS BIBLIOGRÁFICAS}

1. Ferlay J, Bray F, Pisani P, Parkin DM. GLOBOCAN 2002: Cancer Incidence. Mortality and Prevalence Worldwide. IARC CancerBase No. 5, version 2.0., Lyon: IARC Press 2004.

2. Pouratian N, Schiff D. Management of low grade glioma. Curr Neurol Neurosci Rep 2010; 10: 224-31. Doi:10.1007/s11910010-0105-7. 
3. Louis D, Ohgaki H, Wiestler O, Cavenee W, editors. Word Health Organization Classification of Tumors of the Central Nervous System (4d Edition. Acta Neuropathol 2007; 114:97-109. Doi:10.1007/s00401007-0243-4

4. Piepmeir JM. Current concepts in the evaluation and management of WHO grade gliomas. J Neuroncol 2009; 92, 253-9. Doi:10.1007/s11060-009-9870-z

5. Grier JT \& Batchelor T. Low grade gliomas in adults. The oncologist 2006; 11: 681693.

6. Pignatti $F$, van den Bent $M$, Curran $D$, Debruyne C, Sylvester R, Therasse P, et al. Prognostic factors of survival in adults patients with cerebral low -grade gliomas. [abstract]. J Clin Oncol 2002; 20, 2076-84. Doi:10.1200/JCO.2002.08.121

7. Chang E, Smith J, Chang S, Lamborn K, Prados M, Butowski N, et al. Preoperative prognostic classification system for hemispheric low-grade glioma in adults. J Neurosurg 2008; 109: 817-24. Doi:10.3171/ JNS/2008/109/11/0817

8. Meyers C, Hess K, Alfred W, Levin VA. Cognitive function as a predictor of survival in patients with recurrent malignant glioma. J Clin Oncol 2000; 18 (3): 646-50.

9. Taphoorn MJ y Klein M. Cognitive deficits in adult patients with brain tumors. Lancet Neurol 2004; 3:159-68. Doi:10.1016/ S1474-4422(04)00680-5

10. Whittle IR. The dilemma of low-grade glioma. J Neurol Neurosurg Psychiatry 2004; 75(Suppl 2):ii31-ii36. Doi:10.1136/ jnnp.2004.040501

11. Yeh SA, Lee TC, Chen HJ, Lui CC, Sun LM, Wang CJ et al. Treatment outcomes and prognostic factors of patients with supratentorial low-grade oligodendroglioma. Int J Radiat Oncol Biol Phys 2002; 54:1405-9. Doi:10.1016/S0360-3016(02)03053-5

12. Recht LD, Lew R, Smith TW. Suspected low-grade glioma: is deferring treatment safe? [abstract]. Ann Neurol 1992; 31:431436. Doi:10.1002/ana.410310413
13. Shafqat S, Hedley-Whyte ET, Henson JW. Age-dependent rate of anaplastic transformation in low-grade astrocytoma. [abstract]. Neurology 1999; 52: 867-9.

14. Shaw EG, Scheithauer BW, O'Fallon JR, Davis DH. Mixed oligoastrocytomas: A survival and prognostic factor analysis. Neurosurgery 1994; 34: 577-82; discussion 582. Doi:10.1097/00006123-199404000-00002

15. Wessels PH, Weber WE, Raven G, Ramaekers FC, Hopman AH, Twijnstra A. Supratentorial grade II astrocytoma: Biological features and clinical course. Lancet Neurol 2003; 2: 395-403. Doi:10.1016/S14744422(03)00434-4

16. Olson JD, Riedel E, DeAngelis LM. Longterm outcome of low-grade oligodendroglioma and mixed glioma. [abstract]. Neurology 2000; 54:1442-8.

17. Lebrun C, Fontaine D, Ramaioli A, Vandenbos $\mathrm{F}$, Chanalet $\mathrm{S}$, Lonjon $\mathrm{M}$, et al. Longterm outcome of oligodengrogliomas. Neurology 2004; 62:1783-1787.

18. Reijneveld JC, Sitskoorn MM, Klein M, Nuyen J, Taphoorn JM. Cognitive status and quality of life in patients with suspected versus proven low grade gliomas. Neurology 2001; 56, 618-23.

19. Sanz A, Olivares ME, Barcia JA. Aspectos neuropsicológicos en pacientes diagnosticados de tumores cerebrales. Clin Salud 2011; 22 (2): 139-55.

20. Ruge M, Ilmberger J, Tom JC, Kreth FW. Health related quality of life and cognitive functioning in adult patients with supratentorial WHO grade II glioma: Status prior to therapy. J Neurooncol 2011; 103: 129-36. Doi:10.1007/s11060-010-0364-9

21. Yoshii $Y$, Tominaga D, Sugimoto K, Tsuchida Y, Hyodo A, Yonaha H, Kushi S. Cognitive function of patients with brain tumor in pre- and postoperative stage. Surg Neurol 2008; 69: 51-61. Doi:10.1016/j. surneu.2007.07.064

22. Reyes Botero, GA. Gliomas del adulto: acercamiento al diagnóstico y tratamiento. Acta Neurol Colomb 2009; 25 (1): 34-41. 
23. Sheline GE, William WW, Smith V. Therapeutic irradiation and brain injury. Oncol Intelligenc 1980; 6: 1215. Doi:10.1016/0360-3016(80)90175-3

24. Armstrong C, Hunter JV, Ledakis GE, Cohen $\mathrm{B}$, Tallent EM, Goldstein $\mathrm{BH}$, et al. Late cognitive and radiographic changes related to radiotherapy. Initial prospective findings. Neurology 2002; 59: 40-8.

25. Postma TJ, Klein M, Verstappen CC, Bromberg JE, Swennen M, Langendijk JA, et al. . Radiotherapy induced cerebral abnormalities in patients with low grade glioma. Neurology 2002; 59, 121-3.

26. Surma-aho O, Niemela M, Vilkki J, Kouri M, Brander A, Salonen O, et al. Adverse long-term effects of brain radiotherapy in adults low-grade glioma patients. Neurology 2001; 56: 1285-90.

27. Brown PD, Buckner JC, Uhm JH, Shaw EG. The neurocognitive effects of radiation in adult low grade glioma patients. J Neurooncol 2003; 5: 161-7. Doi:10.1215/ S1152851702000431

28. Byrne TN. Cognitive sequelae in brain tumor treatment. Curr Opin Neurol 2005; 18: 662-6. Doi:10.1097/01. wco.0000191511.52745.99

29. Van den Bent MJ, Wefel JS, Schiff D, Taphoorn $M$, Jaeckle $K$, Junck $L$, et al. Response assessment in neuro-oncology (a report of the RANO group): Assessment of outcome in trials of diffuse low-grade gliomas. Lancet Oncol 2011; 12 (6)0, 583-93. Doi:10.1016/S1470-2045(11)70057-2

30. Taphoorn MJ. Neurocognitive sequelae in the treatment of low-grade gliomas. Semin Oncol 2003; 30(6 Suppl 19): 45-8. Doi:10.1053/j.seminoncol.2003.11.023

31. Soffietti R, Baumert BG, Bello $L$, von Deimling $A$, Duffau $H$, Frénay $M$, et al. Guidelines on management of low-grade gliomas: Report of an EFNS-EANO Task Force. Eur J Neurol. 2010; 17(9):1124-33. Doi:10.1111/j.1468-1331.2010.03151.x

32. Armstrong $\mathrm{CL}$, Corn BW, Ruffer JE, Pruitt AA, Mollman JE, Phillips PC.
Radiotherapeutic effects on brain function: Double dissociation of memory systems. Neuropsychiatr Neuropsychol Behav Neurol 2000; 13: 101-1.

33. Taphoorn MJ, Klein Schiphorst A, Snoek FJ, Lindeboom J, Wolbers JG, Karim Ab, et al. Cognitive functions and quality of life in patients with low grade gliomas: The impact of radiotherapy. Ann Neurol 1994; 36: 48-54. Doi:10.1002/ana.410360111

34. Correa CC, De Angelis LM, Shi W, Thaler $\mathrm{H}$, Lin M, Abrey LE. Cognitive functions in low grade gliomas: Disease and treatment effects. J Neurooncol 2007; 81: 175-84. Doi:10.1007/s11060-006-9212-3

35. Correa CC, Shi W, Thaler H, Cheung A, De Angelis LM, Abrey LE. Longitudinal cognitive follow up in low grade gliomas. J Neurooncol 2008; 86: 321-7. Doi:10.1007/ s11060-007-9474-4

36. Lezak MD. Neuropsychological Assessment (3rd Edition). New York: Oxford University Press. 1995.

37. Armstrong C, Mollman, J, Corn BW, Alavi J, Grossman M. Effects of radiation therapy on adult brain behavior: Evidence for a rebound phenomenon in a phase 1 trial. Neurology 1993; 43: 1961-5.

38. Armstrong C, Ruffer J, Corn B, DeVries K, Mollman J. Bi phasic patterns of memory deficits following moderate-dose partialbrain irradiation: Neuropsychologic outcome and proposed mechanisms. J Clin Oncol 1995; 13: 2263-71.

39. Armstrong C, Stern CH, Corn BW. Memory performance used to detect radiation effects on cognitive functioning. Appl Neuropsychol 2001; 8 (3): 129-39. Doi:10.1207/S15324826AN0803_1

40. Gregor A, Cull A, Traynor E, Stewart M, Lander F, Love S. Neuropsychometric evaluation of long-term survivors of adult brain tumors: relationship with tumor and treatment parameters. Radiat Oncol 1996; 41: 55-9. Doi:10.1016/S0167-8140(96)01782-3

41. DeAngelis LM, Delattre JY, Posner JB. Radiation-induced dementia in patients cu- 
red of brain metastases. Neurology 1989; 39:789-96.

42. Klein M, Heimans JJ, Aaronson NK, Van der Ploeg, HM, Grit J, Muller M, et al. Effect of radiotherapy and other treatment related factors on mild term to long term cognitive squeals in low grade gliomas: A comparative study. Lancet 2002; 360: 1361-8. Doi:10.1016/S0140-6736(02)11398-5

43. Douw L, Klein M, Fagel SA, Van den Heuvel J, Taphoorn MT, Aaronson NK, et al. Cognitive and radiological effects of radiotherapy in patients with low-grade glioma: long term follow up. Lancet Neurol 2009; 8: 810-8. Doi:10.1016/S14744422(09)70204-2

44. Packer R, Mehta M. Neurocognitive sequeale of cancer treatment. Neurology 2002; 59: 8-10.

45. Kiebert GM, Curran D, Aaronson NK, BoIla M, Menten J, Rutten EH, et al. Quality of life after radiation therapy of cerebral low-grade gliomas of adults: results of a randomized phase III trial on dose response (EORTC trial 22844). EORTC Radiotherapy Co-operative Group. Eur J Cancer 1998; 34: 1902-9. Doi:10.1016/S09598049(98)00268-8

46. Sarkissian V. The sequale of cranial irradiation on human cognition. Neurosci Lett 2005; 382: 118-23. Doi:10.1016/j.neulet.2005.02.068

47. Nieto MA, Sánchez MP, Barroso J, Olivares T, Hernández MA. Alteraciones cognitivas en etapas iniciales de la esclerosis múltiple y su relación con el estado de ánimo, variables demográficas y clínicas. Psicothema 2008; 20 (4): 583-8.

48. De Quesada ME, Reyes M, Díaz G. Alteraciones cognitivas en pacientes con hipertensión arterial no complicada. Vitae Academia Biomédica Digital, $n^{\circ}$ 34. [En línea] 2008 [Acesso 1 de octubre de 2011] Disponible en: http://vitae.ucv.ve/index_pdf. php? module $=$ articulo_pdf $\& n=1285 \& r v=42$

49. Vicario A, Martínez C. Evaluación del daño funcional del cerebro en pacientes hipertensos: empleo del Examen Cognitivo Mínimo. Rev Fed Arg Cardiol 2007; 36: 146-51.

50. Uribe M, González W. Leucoencefalopatías tóxicas. En Asociación Colombiana de Neurología, editores. Guía Neurológica 5. p.: 31-41. http://www.acnweb.org/ guia/ g5cap4.pdf.

51. Hammack JE, Shaw E, Ivnik R, Arusell R, Novotny $\mathrm{P}, \mathrm{O}^{\prime}$ Fallon J. Neurocognitive function in patient receiving radiation therapy for supratentorial low glioma: A North Central Cancer Treatment Group (NCCTG) prospective study. [abstract]. Proc. Am Soc Clin Oncol 1995; 14: 151.

52. Vigliani MC, Sichez N, Poisson M, Delattre JY. A prospective study of cognitive functions following conventional radiotherapy for supratentorial gliomas in young adults: 4-year results. Int J Radiat Oncol Biol Phys 1996; 35 (3): 527-33. Doi:10.1016/S0360-3016(96)80015-0

53. Laack N, Brown $P$, Ivnik $R$, Furth $A$, Ballman K, Hammack J, et al. Cognitive function after radiotherapy for supratentorial low-grade glioma: A North Central Cancer Treatment Group prospective study. Int J Radiat Oncol Biol Physics 2005, 63 (4): 1175-83. Doi:10.1016/j.ijrobp.2005.04.016

54. Brown P, Buckner JC, O'Fallon J, Iturria $\mathrm{N}$, Brown CA, O'Neill BP, et al. Effects of radiotherapy on cognitive function in patients with low grade glioma measured by the Folstein Mini Mental State Examination. J Clin Oncol 2003; 21: 2519-24. Doi:10.1200/JCO.2003.04.172

55. Torres IJ, Mundt AJ, Sweeney PJ, LlanesMacy S, Dunaway L, Castillo $M$ et al. A longitudinal neuropsychological study of partial brain radiation in adults with brain tumors. Neurology 2003; 60:1113-8.

56. Stroop, JR. Studies of interference in serial verbal reactions. J Exp Psychol 1935;18: $643-62$.

57. Benton AL, Hamsher K. Multilingual Aphasia Examination, 2a ed. Iowa City: De- 
partment of Neurology and Psychology. University of lowa. 1989.

58. Van der Elst W, Van Boxtel MP, Van Breukelen GJ, Jolles J. The Concept Shifting Test: adult normative data. Psychol Assess 2006; 18 (4):424-32. Doi: 10.1037/10403590.18.4.424

59. Wechsler D. Wechsler Adult Intelligence Scale-Third Edition. San Antonio: The Psychological Corporation; 1997.

60. Wechsler D. Wechsler Intelligence Scale for children. New York: Psychological Corp, 1949.

61. Heaton RK. Wisconsin Card Sorting Test Manual. Odessa, FL: Psychological assessment resource, Inc.

62. Spreen O, Benon AL. Neurosensory Center Comprehensive Examination for Apahasia. Victoria B.C. Neuropsychological Laboratory Dept. of Psychology. University of Victoria. 1969.

63. Brickenkamp R, Zillmer E. The $\mathrm{d} 2$ Test of Attention. Seattle: Hogrefe \& Huber Publishers.1998.

64. Army Individual Test Battery. Manual of directions and DC, EEUU: War Department, Adjutant General's Office. 1944.

65. Doverspike D, Cellar D, Barrett, GV. The auditory selective attention test: A review of field and laboratory studies. Educ Psychol Meas 1986: 46: 1095-104. Doi:10.1177/001316448604600432

66. Armstrong C. Selective versus sustained attention a continuous performance test revised. Clin Neuropsychol 1997; 11: 18-33. Doi:10.1080/13854049708407026

67. McKenna P, Warrington EK. Graded Naming Test. Windsor: Nelson, 1983.

68. Rosvold HE, Mirsky AF, Sarason I, Bransome Jr. ED, Beck LH. A Continuous performance test of brain damage. J Consult Psychol 1956; 20: 343-50. Doi:10.1037/ h0043220

69. Gauthier L, Dehaut F, Joanette Y. The bells: A quantitative and qualitative test for visual neglect. Inter J Clin Neuropsychol 1989; 11: 49-54.
70. Rey A. L'examen clinique en psychologie. Paris: Presse Universitaire de France. 1958.

71. Delis DC, Kramer JH, Kaplan E, Ober BA. The California Verbal Learning Test. San Antonio, TX: Psychological Corporation. 1987.

72. Hannay HJ, Levin HS. Selective Reminding Test: An examination of the equivalence of four forms. J Clin Exp Neuropsychol 1985; 7(3): 251-63. Doi:10.1080/01688638508401258

73. Grossman M, Armstrong C, Onishi K, Thompson H, Schaefer B, Robinson K, et al. Patterns of cognitive impairment in relapsing-remitting and chronic-progressive multiple sclerosis. Neuropsychiatry Neuropsychol Behav Neurol 1994, 7:194-210.

74. Benton $A L$, Fogelm L. Test de rétention visuelle: normes revues et completées. Rev Psychol Appl 1961; 11: 75-7.

75. Benton L, Varney NR, Hamsher K. Visuospatial judgment: A clinical test. Arch Neurol 1978; 35: 364-7.

76. Osterrieth P A. Le test de copie d'une figure complex: contribution a l'etude de la perception et de la memoire. Arch Psychol (Geneve) 1944; 30: 296-356.

77. Lindeboom J. Measurement of anterograde amnesia. [abstract]. J Clin Exp Psy 1989; 11: 345.

78. Rao, SM. A manual for the brief repeatable battery of neuropsychological test in multiple sclerosis. New York: National multiple sclerosis society, 1990.

79. Benton A, Hamsher K, Varney NR, Spreen O. Judgment of Line Orientation. New York: Oxford University Press, 1983.

80. Schenkenberg T, Bradford DC, Ajax ET. Line bisection and unilateral visual neglect in patients with neurological impairment. Neurology 1980; 30: 509-17.

81. Benton L, Varney NR, Hamsher K. Visuospatial judgment: A clinical test. Arch Neurol 1978; 35:364-7. Doi:10.1016/00283932(78)90049-0

82. Money J, Alexander D, Walker HT Jr. A stantarized Road Map test of direction sense. Baltimore: Johns Hopkins Press: 1965. 
83. Warrington EK, James M. Visual Object and Space Perception Battery (VOSP). Thames Valley Test Company, Bury St Edmunds 1991.

84. Burgess PW, Shallice T. The Hayling and Brixton Tests. Bury St. Edmunds: Thames Valley Test Company, 1997.

85. Schmand B, Bakker D, Saan R, Louman J. The Dutch Reading Test for Adults: A measure of premorbid intelligence level. Tijdschr Gerontol Geriatr. 1991; 22(1):159. [Article in Dutch].

86. Nelson HE, Willison JR. The National Audit Reading Test. Edt. Test Manual, 1991. NFER-Nelson, Windsor.

87. Zachary, R. A. Shipley Institute of Living Scale: Revised Manual. J Psychol 1986; 9: 371-7.

88. Jolles J, Houx PJ, Van Boxtel MPJ, Ponds RWHM. Maastricht Aging Study: Determinants of cognitive aging. Maastricht, The Netherlands: Neuropsych Publishers. 1995. Doi:10.1016/S0197-4580(00)83163-2

89. Shimoyama I, Ninchoji T, Uemura K. The Finger-Tapping Test. A quantitative analysis. Arch Neurol 1990; 47(6):681-4.

90. Gronwall, DMA. Paced auditory serialaddition task: A measure of recovery from concussion. Percept Mot Skills, 1977; 44: 367-73. Doi:10.2466/pms.1977.44.2.367

91. Leslie SC, Davidson RJ, Batey OB. Purdue Pegboard performance of disabled and normal readers: Unimanual versus bimanual differences. Brain Lang 1985; 24: 359-69. Doi:10.1016/0093-934X(85)90140-3

92. Folstein MF, Folstein SE, McHugh PR. "Mini-mental state". A practical method for grading the cognitive state of patients for the clinician. J Psychiatr Res 1975; 12: 18998. Doi:10.1016/0022-3956(75)90026-6

93. Marsh JC, Gielda BT, Herskovic AM, Abrams R. Cognitive sparing during the administration of whole brain radiotherapy and prophylactic cranial irradiation: Current concepts and approaches. J Oncol 2010. Published online 2010 June 27. Doi: 10.1155/2010/198208.

94. Tirapu-Ustárroz J, Luna-Lario P, HernáezGoñi P, García-Suescun I. Relación entre la sustancia blanca y las funciones cognitivas. Rev Neurol 2011; 52: 725-42.

95. Brown PC, Cerhan JH. Same, better, or worse? Neurocognitive effects of radiotherapy for low grade gliomas remain unknown. Lancet 2009; 8: 779-81. Doi:10.1016/ S1474-4422(09)70205-4

96. Blazquez JL, González B, Paul N. Evaluación Neuropsicológica. En: Tirapu-Ustárroz J, Rios M, Maestú F, editores. Manual de Neuropsicología. 2a edición. Barcelona: Viguera Editores, SL. p. 33-56.

97. Fox S, Mitcheli S, Booth-Jones, M. Cognitive impairment in patients with brain tumors: Assessment and intervention in the clinic setting. Clin J Oncol Nurs 2006; 10 (2): 16976. Doi: 10.1188/06.CJON.169-176 